\title{
Brain potentials in affective picture processing: covariation with autonomic arousal and affective report ${ }^{\text {th }}$
}

\author{
Bruce N. Cuthbert ', Harald T. Schupp ${ }^{2}$, \\ Margaret M. Bradley, Niels Birbaumer ${ }^{3}$, Peter J. Lang* \\ NIMH Center for the Study of Emotion and Attention (CSEA), University of Florida, \\ Box 100165 HSC, Gainesville, FL 32610-0165, USA
}

Received 13 August 1998; received in revised form 19 November 1999; accepted 19 November 1999

\begin{abstract}
Emotionally arousing picture stimuli evoked scalp-recorded event-related potentials. A late, slow positive voltage change was observed, which was significantly larger for affective than neutral stimuli. This positive shift began 200-300 ms after picture onset, reached its maximum amplitude $\approx 1 \mathrm{~s}$ after picture onset, and was sustained for most of a 6-s picture presentation period. The positive increase was not related to local probability of content type, but was accentuated for pictures that prompted increased autonomic responses and reports of greater affective arousal (e.g. erotic or violent content). These results suggest that the late positive wave indicates a selective processing of emotional stimuli, reflecting the activation of motivational systems in the brain. (C) 2000 Elsevier Science B.V. All rights reserved.
\end{abstract}

Keywords: Emotion; Attention; Arousal; Event-related potentials; Slow potentials

\footnotetext{
is Part of this data was presented at the 35th Meeting of the Society for Psychophysiological Research, Toronto, Canada (see Cuthbert et al. (1995), abstract).

* Corresponding author.

E-mail address: langlab@nersp.nerdc.ufl.edu (P.J. Lang)

${ }^{1}$ Present address: National Institute of Mental Health, Rockville, MD, USA.

${ }^{2}$ Present address: University of Greifswald, Germany.

${ }^{3}$ Present address: University of Tübingen, Germany.
} 


\section{Introduction}

The aim of this experiment is assess the brain's reactivity to emotional pictures by recording event-related potentials (ERPs) and assessing their relationship to evaluative, somatic, and autonomic measures of affect. The perceptual processing of pictures, in terms of the degree of attentive engagement, is presumed to be determined in considerable part by their motivational significance. Following Konorski (1967), most pleasant affects are held to be associated with the brain's appetitive motivation system; unpleasant affects with defensive motivation. Reports of increased arousal and greater magnitude of sympathetically mediated responses (e.g. sweat-gland activity) index increased activation of motive systems (appetitive, defensive, or both), and a commensurate greater intensity or vigor of the affective response (Lang et al., 1990, 1997, 1998; see also Cacioppo and Berntson, 1994; Davidson, 1995).

For a representative sample of photographic stimuli in the International Affective Picture System (IAPS; SEA, 1999; Lang et al., 1999), participants' bi-polar ratings of affective valence (pleasure-displeasure) and arousal (calm-aroused) reflect this motivational foundation. Thus, judgments of increasing pleasure, and increasing displeasure, are in general both positively correlated with reports of greater arousal (suggesting that arousal reports index the level of both appetitive and defensive motive intensity; (Lang et al., 1997). Furthermore, behavioral and physiological responses to pictures support this conception, with individual measures co-varying either with system engagement (valence) or with motive intensity (arousal) (for example, see Greenwald et al., 1989; Bradley et al., 1990; Lang et al., 1993). Thus, facial actions during picture viewing (as measured by electromyographic activity of corrugator and zygomatic muscles) are associated with reports of pleasure or displeasure - more corrugator tension is found during viewing of unpleasant pictures and more zygomatic activity during pleasant pictures. Among autonomic responses, heart rate co-varies most strongly with affective valence. On the other hand, skin conductance increases with the judged affective arousal (motivational intensity) of both pleasant and unpleasant pictures. Memory for specific pictures and viewing time also correlate with arousal (Bradley et al., 1992), suggesting that there is greater attention at encoding for any strong emotional stimuli, regardless of which motive system is active.

In the cognitive literature (Kok, 1997), stimulus evaluation, target identification, and other recognition processes are associated with positive ERP components (e.g. P3). Several research groups have also reported enhanced late positive potentials, later in the waveform ( $400 \mathrm{~ms}$ and beyond), that are evoked specifically by emotional pictures (Johnston et al., 1986; Naumann et al., 1992; Diedrich et al., 1997; Palomba et al., 1997). Similar results have been obtained in studies of subjects viewing affective facial expressions (Orozco and Ehlers, 1998) or faces that vary in attractiveness (Johnston and Oliver-Rodríguez, 1997). In all of this research, the late positive waveform generally begins in the region of the P3 - the classic index of attention, recognition, and stimulus probability (Donchin and Coles, 1988) suggesting an association between these ERP measures. 
Theory regarding more sustained slow waves (of $1 \mathrm{~s}$ or more) has traditionally been more concerned with negative than positive going waveforms (see reviews by Birbaumer et al., 1990; Rösler et al., 1997). However, several recent studies indicate that positive centro-parietal slow waves are sensitive to manipulations in the perceptual domain (Ritter and Ruchkin, 1992). Thus, Ruchkin et al. (1988) proposed that, while slow negative waves may reflect the brain's higher-order conceptual activity, positive slow waves vary with perceptual operations and memory storage. Consistent with this view, Kok (1997) assumes that positive slow waves and P3s reflect similar processes. These views and findings suggest that enhanced slow waves might be expected during picture viewing. Furthermore, they should be specifically enhanced for emotional stimuli, reflecting a greater allocation of perceptual processing resources to motivationally relevant input.

An important concern for ERP studies of emotion concerns validation of the instigating cues to affect. Self-report of feeling, physiological reactivity (autonomic and somatic) and behavioral measures are all relevant to the description of an emotional response (Mandler et al., 1961; Lang, 1989). The validation of affective input requires their assessment. If ERPs are truly affective in origin, i.e. motivationally determined, it must be shown that the evoking stimuli have a systematic relationship with emotion's multiple output measures.

\section{The research problem}

The present experiment evaluates late positive potentials and sustained positive slow waves during affective stimulus exposure, simultaneously recording peripheral physiological changes and affective reports that have been shown to be associated with emotional reactivity (e.g. Lang et al., 1993). Participants view a series of pictures, selected on the basis of IAPS valence ratings (Lang et al., 1999) into equal numbers of neutral, pleasant, and unpleasant stimuli, presented randomly. According to the oddball hypothesis, P3-like ERPs are enhanced to rare stimuli (i.e. stimuli with a low subjective probability). Thus, it is important that the local probability is the same for the three content types. Furthermore, the emotionally arousing pictures collectively (pleasant and unpleasant) are twice as frequent as the neutral pictures, which are all rated low in arousal. If participants conceive the pictures to fall into two groups (i.e. emotionally arousing versus neutral stimuli), the P3-oddball hypothesis predicts that the more rare neutral pictures should show the larger ERP positivity. Thus, the construction of the picture set (if it can be construed as biased) works against support of the motivation hypothesis.

The overall aim of the experiment was to determine if the ERP late positive potential and the sustained positive slow wave that occurs in response to picture stimuli is determined by the participant's emotional engagement with the stimulus. In analyzing the data, several specific issues were addressed.

1. Do neutral, pleasant, and unpleasant pictures differ systematically in affective ratings, facial muscle change, and autonomic response (heart rate and skin conductance)? 
2. Are late positive potentials in the ERP and the slow wave greater for emotional than neutral picture stimuli, and is the slow wave more sustained?

3. Does the overall ERP waveform and the relationship between the ERP response to emotional and neutral pictures differ according to electrode site?

4. When separately analyzed for pleasant and unpleasant pictures, is ERP late positivity greater for pictures rated higher in arousal?

5. Based on factor analysis of all response to these picture stimuli, does ERP late positivity load on the same factor as other measures of emotional arousal?

\section{Method}

\subsection{Subjects}

Thirty-seven (14 female) volunteers (approximate age range, 18-24 years) from the University of Florida Introductory Psychology course participated as part of a class requirement. Due to computer failure, peripheral physiological data were lost for one participant, and electrocortical data for two participants.

\subsection{Stimulus materials}

Fifty-four pictures were taken from IAPS (SEA, 1999; see Lang et al., 1999), depicting 18 unpleasant events (e.g. spiders, mutilations, etc.), 18 pleasant events (e.g. attractive infants, opposite sex nudes, etc.) and 18 neutral events (e.g. neutral faces household objects, etc. $)^{4}$. The content groups were selected such that there was almost no overlap in IAPS normative affective valence ratings, i.e. the three stimulus contents were distinct and representative of affect type: mean valence (nine-point scales, pleasant high) for pleasant pictures, 7.4; neutral pictures, 5.0; unpleasant pictures, 2.7. All neutral pictures had lower standard emotional arousal ratings (mean $=2.9$ ) than the pleasant $($ mean $=5.8)$ and unpleasant $($ mean $=6.4)$ pictures. The distributions of arousal ratings for pleasant and unpleasant pictures were overlapping. Both contents were anchored at the high end by similar high arousal exemplars; because of the natural picture distribution of the IAPS, the low arousal end of the unpleasant pictures range was somewhat less extended than for pleasant pictures ${ }^{5}$.

\footnotetext{
${ }^{4}$ The IAPS slide numbers were as follows: pleasant, 1600, 2080, 2250, 4180, 4210, 4250, 4290, 4310, $4470,4500,4520,4550,4490,4650,4660,4680,7200,7330,7350,8030,8080$, 8200, 8510; neutral, 2190, $2200,5500,7000,7010,7020,7050,7080,7090,7100,7130,7150,7160,7170,7180,7500,7550,7700$; negative, 1070, 1090, 1120, 1300, 2120, 3000, 3010, 3100, 3130, 3150, 3530, 6020, 6190, 6200, 6230, 6370, 9040, 9490. The IAPS and technical manuals can be obtained from the corresponding author.

${ }^{5}$ In the Cartesian space defined by affective valence and arousal measures, pleasant and unpleasant IAPS pictures show different distributions (Bradley and Lang, in press). Thus, for example, unpleasant pictures show a high positive correlation between valence and arousal (0.55) that is particularly pronounced for female subjects $(0.71)$. For pleasant pictures, this correlation is somewhat lower overall (0.46), but palpably higher for males (0.64). The difference in these natural distributions prevents exact balancing of arousing stimuli for valence, in a way that would still include the strongest stimuli of both types. Of some importance to emotion theory, these differences further imply that the organization of pleasant and unpleasant emotion is not symmetrical.
} 
The pictures were presented as color slides, arranged in three blocks, such that each block contained six positive, six neutral, and six negative pictures. Three orders of picture presentation were constructed such that, across participants, each block was seen equally often in the first, second, or third position. In addition, picture order within blocks was varied, such that across participants, each picture was seen equally often in the first, middle or last portion of each block. Each order was presented to approximately one-third of the participants.

\subsection{Procedure}

After arrival at the laboratory, participants read and signed an informed consent form and filled out several questionnaires. Participants were then seated in a recliner in a small, sound-attenuated, dimly lit room, and electrodes were attached. The participant was told that a series of slides would be presented and that he/she should attend to each picture the entire time it appeared on the screen. The slide image was projected for $6 \mathrm{~s}, \approx 1.9 \mathrm{~m}$ from the participant's eyes $\left(\approx 25^{\circ}\right.$ of visual angle). In order to promote a stable mental set, the participant was asked to try to maintain the image of the slide for a short period after its presentation (terminated by a soft tone). The participant was then asked to judge their emotional reactions while viewing the picture on bi-polar scales measuring affective valence and arousal. A variable interval $(12-18 \mathrm{~s})$ occurred between each trial ${ }^{6}$. A 1 min resting baseline was initiated at the beginning of the experiment to facilitate laboratory adaptation, and two neutral pictures served as practice trials.

\subsection{Psychophysiological recording procedures}

Peripheral and electroencephalogram (EEG) signals were recorded from $3 \mathrm{~s}$ before slide onset until $1 \mathrm{~s}$ after the end of the post-picture period (only the data for picture viewing are reported here). The EEG was recorded from nine sites using Nihon-Kohden amplifiers according to the international 10-20 system. $\mathrm{Ag} / \mathrm{AgCl}$ electrodes were placed at $\mathrm{F} 3, \mathrm{Fz}, \mathrm{F} 4, \mathrm{C} 3, \mathrm{Cz}, \mathrm{C} 4, \mathrm{P} 3, \mathrm{Pz}, \mathrm{P} 4$, and both mastoids. EEG signals were digitized on a Macintosh computer with LABVIEW software; all channels were recorded using $\mathrm{Cz}$ as a reference, digitally converted off-line to a linked-mastoid reference. In order to shorten the time lag between EEG channels during data sampling, the actual sampling rate was $1250 \mathrm{~Hz}$, decimated off-line to a $125 \mathrm{~Hz}$ sampling rate. The time constant was set at $10 \mathrm{~s}$ and the high-frequency cut-off was $35 \mathrm{~Hz}$. Vertical and horizontal eye movements were recorded using miniature $\mathrm{Ag} / \mathrm{AgCl}$ electrodes and the same frequency range as the EEG.

\footnotetext{
${ }^{6}$ Acoustic probes $(50 \mathrm{~ms} ., 95 \mathrm{~dB}(\mathrm{~A}))$ were presented during or after some trials and blinks were recorded from electrodes over the left orbicularis oculi muscle. These data were reported in Schupp et al. (1997).
} 
A IBM-compatible computer and VPM software (Cook, 1997) was used for timing and stimulus control, as well as acquisition of autonomic and somatic signals, and affective ratings. Recording of peripheral measures was identical to that described in detail in Lang et al. (1993). Briefly, the electrocardiogram (EKG) was recorded with a Coulbourn S75-01 bioamplifier, using electrodes on the left and right forearms; a Schmitt trigger was used to interrupt the computer at the occurrence of each $\mathrm{R}$ wave of the EKG, and record inter-beat intervals to the nearest millisecond. Skin conductance was recorded in microsiemens with a Coulbourn S71-22 bioamplifier, from electrodes affixed to the hypothenar eminence of the left palm. Activity over the left corrugator muscle area was recorded with a Coulbourn S75-01 bioamplifer, with a bandpass of $90-1000 \mathrm{~Hz}$, and rectified and integrated with a contour following integrator (time constant $500 \mathrm{~ms}$ ).

Valence and arousal ratings were obtained using a graphic, interactive, computerized version of the Self-Assessment Manikin (SAM; Lang, 1980; Hodes et al., 1985; Cook, 1997). SAM is scaled from 0 to 20 : it was developed as a user-friendly alternative to the semantic differential, with which it is highly correlated (Bradley and Lang, 1994). Imagery vividness ratings were also obtained by using a line rating with the endpoints labeled 'not vivid at all' and 'as vivid as possible'.

\subsection{Data reduction and analysis}

All individual EEG trials that were off-scale at any time during the $16 \mathrm{~s}$ recording epoch were excluded from further analysis; on average, $10.2 \%$ of the trials were thus excluded. To correct for vertical and horizontal ocular artifacts, an eye movement artifact correction procedure (Gratton et al., 1983, revised by Miller et al., 1988) was applied to the EEG recordings including $1 \mathrm{~s}$ baseline, picture and post-picture periods, and $1 \mathrm{~s}$ post-baseline. Following eye movement correction, ERP grand average waveforms were calculated separately for each valence category and electrode site, referenced to a $120 \mathrm{~ms}$ baseline preceding picture onset. Visual inspection served to determine areas of interest. As shown in Fig. 1, event-related peaks, especially $\mathrm{N} 2$ and $\mathrm{P} 3$, were not pronounced, and area measures seemed to be more appropriate for these waveforms. A window from 200 to $300 \mathrm{~ms}$ captured the P2 and N2 peaks, which had mean latencies of 210 and $274 \mathrm{~ms}$, respectively. An area from 300 to $400 \mathrm{~ms}$ captured the P3 peak, with a mean latency of $373 \mathrm{~ms}$. Following the P3, a negative going wave developed, which was most pronounced at fronto-central leads. This broad peak was analyzed by a window from 400 to 700 ms. The window from 700 to $1000 \mathrm{~ms}$ assessed the further transition towards a sustained positive slow wave. The positive slow wave was scored by mean activity in successive $1 \mathrm{~s}$ bins from 1 to $6 \mathrm{~s}$ (i.e. the last $5 \mathrm{~s}$ of the picture).

Inter-beat intervals of the EKG were converted to heart rate in beats per minute, and peak acceleration during the viewing interval was scored. Skin conductance was scored for peak response during viewing; corrugator EMG was expressed as the average change in voltage over the $6 \mathrm{~s}$ viewing interval. All these measures were change scores deviated from a 1 s pre-picture baseline period (see Lang et al. (1993) for details). 
All data (ratings, peripheral physiology, and ERP) were analyzed with a repeated-measures analysis of variance (ANOVA), with a single primary factor of emotion content (pleasant, neutral, and unpleasant); significant main effects ( $\alpha=$ $0.05)$ were followed by contrasts to identify specific differences. In order to provide a careful assessment of temporal ERP effects, separate analyses were conducted for each time epoch during the first second after picture onset; for the remaining $5 \mathrm{~s}$ of

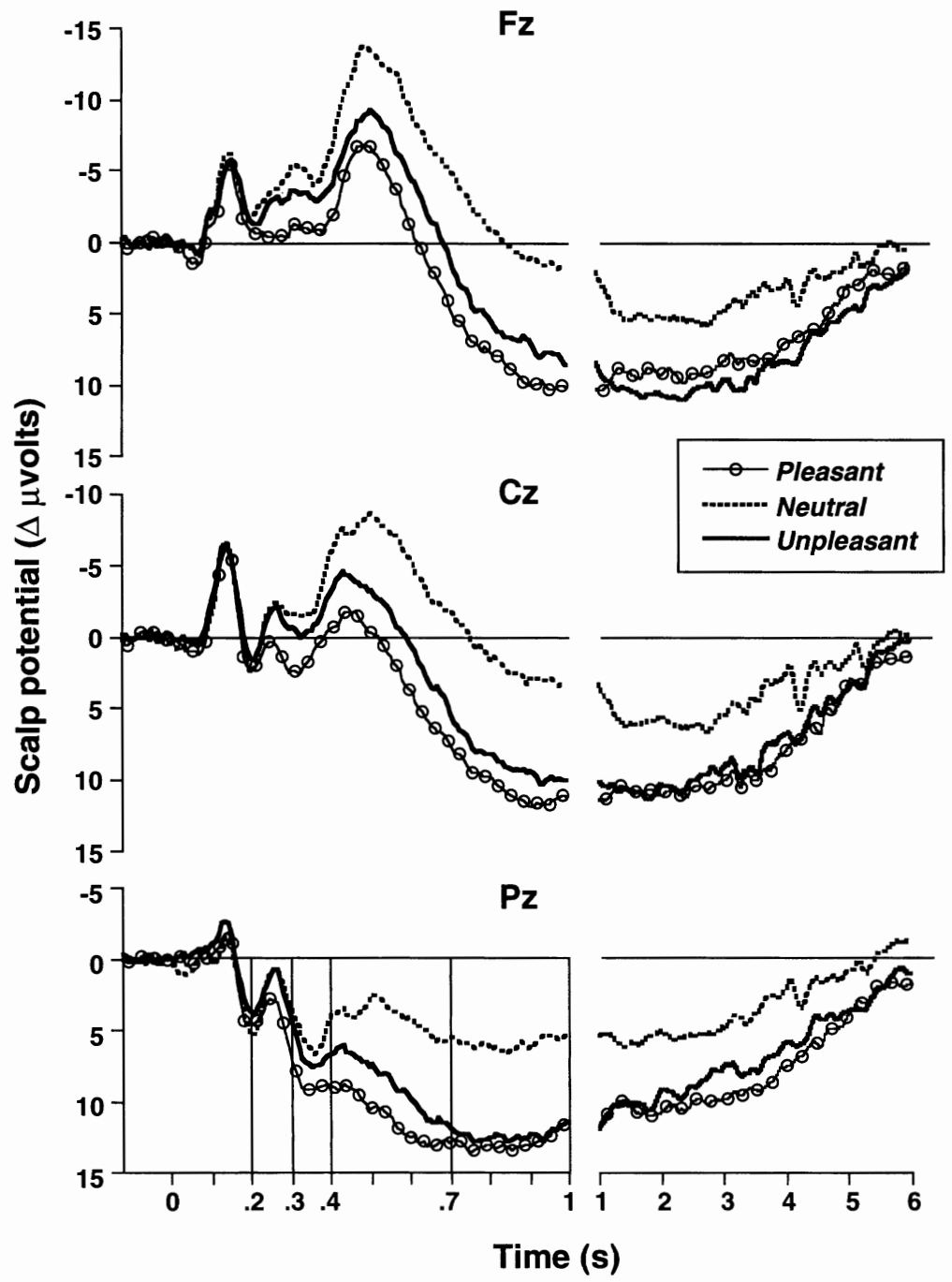

Fig. 1. Stimulus synchronized grand average ERP waveforms for $\mathrm{Fz}, \mathrm{Cz}$, and $\mathrm{Pz}$ electrodes during viewing of affective pictures, separately for each valence category (pleasant, neutral and unpleasant). The left panel illustrates the picture onset potentials on a finer time scale, and the vertical lines at $\mathrm{Pz}$ illustrate the time areas subjected to statistical analysis (i.e. $200-300,300-400,400-700,700-1000 \mathrm{~ms}$ ). The right panel shows the subsequent $5 \mathrm{~s}$ of slow potential change. 
Table 1

Means for valence and arousal ratings, corrugator response, skin conductance response, and heart rate change for pleasant, neutral, and unpleasant pictures ${ }^{\mathrm{a}}$

\begin{tabular}{lccc}
\hline Measures & $\begin{array}{l}\text { Pleasant } \\
\text { pictures }\end{array}$ & $\begin{array}{l}\text { Neutral } \\
\text { pictures }\end{array}$ & $\begin{array}{l}\text { Unpleasant } \\
\text { pictures }\end{array}$ \\
\hline Valence ratings & $16.1(1.9)$ & $10.8(1.4)$ & $5.9(3.1)$ \\
Arousal ratings & $14.5^{\mathrm{a}}(2.8)$ & $6.6(3.1)$ & $14.6^{\mathrm{a}}(2.6)$ \\
Corrugator EMG change $(\mu \mathrm{V})$ & $-0.16(0.88)$ & $0.3(0.66)$ & $0.54(0.93)$ \\
$\begin{array}{l}\text { Skin conductance response, } \\
\quad \text { log }(\mu \text { siemens }+1)\end{array}$ & $0.08(0.09)$ & $0.01(0.03)$ & $0.05(0.08)$ \\
Heart rate peak $(\mathrm{bpm})$ & $3.9^{\mathrm{a}}(2.7)$ & $3.5^{\mathrm{a}}(2.6)$ & $2.2(2.7)$ \\
\hline
\end{tabular}

${ }^{a}$ Valence and arousal ratings were on a $0-20$ scale. Values sharing a letter within a row do not differ significantly in a post-hoc comparison for that response $(P<0.05)$. Standard deviations are presented in parentheses.

the picture, an additional repeated measures factor of time (five $1 \mathrm{~s}$ bins) was included. Two separate sets of analyses were conducted: the midline analysis also included electrode location as a factor (frontal, central, and parietal), while the analysis of left and right leads involved an additional laterality factor. All ANOVAs with more than two factors were conducted using a multivariate test statistic (Wilks' lambda) in order to control for deviations from sphericity.

\section{Results}

\subsection{Autonomic, somatic and self-report measures}

As in Lang et al. (1993), the evaluative, somatic, and autonomic responses obtained in the viewing context reliably discriminated emotional from neutral pictures, and confirmed differences between affective contents. As shown in Table 1 , unpleasant pictures were judged to be significantly less pleasant than neutral pictures, which were in turn rated less positively than pleasant pictures: emotion content, $F(2,33)=124.3, P<0.0001$. Emotional pictures, pleasant and unpleasant, did not differ in arousal reports, but were both judged to be significantly more arousing than neutral pictures: emotion content, $F(2,33)=125.1, P<0.0001$. Unpleasant pictures occasioned significantly greater corrugator muscle activity and greater heart rate deceleration (i.e. a lower peak heart rate) than other picture types: emotion content, $F s(2,33)=3.9$ and $12.1, P<0.05$, respectively. Both emotional picture types prompted significantly larger increases in skin conductance than did neutral pictures, and in this experiment, conductance responses to pleasant pictures were significantly larger than those to unpleasant images: emotion content, $F(2,33)=11.2, P<0.0001$. 


\subsection{Event-related potentials}

The data from frontal $(\mathrm{Fz})$, central $(\mathrm{Cz})$, and parietal sites $(\mathrm{Pz})$, distributed along the anterior-posterior midline of the brain, are presented in Fig. 1. In order to highlight points of interest, the phasic waveform obtained in the first second after picture onset is presented on a finer time scale than the subsequent $5 \mathrm{~s}$ of slow potential change. The general topography of the cortical response included a prominent, early negative peak at $100 \mathrm{~ms}$. and subsequent positive going waves in the regions $200-300$ and $300-400 \mathrm{~ms}$. Following these peaks, a positive slow potential developed which persisted throughout most of the $6 \mathrm{~s}$ picture presentation period; at fronto-central sites, this slow potential was preceded by a prominent negative peak between 400 and $700 \mathrm{~ms}$.

Midline cortical response differences among picture contents began between 200 and $300 \mathrm{~ms}$ after picture onset (Fig. 1), with significantly more positivity during pleasant relative to neutral pictures: contrast, $F(1,34)=10.7, P<0.01$; emotion content, $F(2,33)=5.2, P<0.02$. For the subsequent P3 region of $300-400 \mathrm{~ms}$, pleasant pictures continued to prompt the greatest positivity - significantly more than both neutral and unpleasant stimuli: contrast, $F s(1,34)=28.3$ and $8.6, P<$ 0.01 ; emotion content, $F(2,33)=14.2, P<0.0001$. From 400 to $700 \mathrm{~ms}$, during the large fronto-central negative wave, the response to pleasant pictures was still significantly different from both neutral and unpleasant contents $(F s(1,34)=86.5$ and $8.7, P<0.01$ ), but now unpleasant pictures also prompted a positive-going shift compared with neutral images: contrast, $F(1,34)=24.9, P<0.0001$; emotion content, $F(2,33)=42.5, P<0.0001$. From 700 to $1000 \mathrm{~ms}$, pleasant and unpleasant pictures were not different, and both showed significantly more positivity than neutral: contrast $F s(1,34)=126.2$ and $43.4, P<0.0001$; emotion content $F(2,33)=$ 64.5, $P<0.0001$.

The interaction of emotion content and location was never significant. However, significant main effects of location were found, $F \mathrm{~s}(2,23)=18.9,50.2,131.2$ and 30.8 for areas 200-300, 300-400, 400-700, 700-1000 ms, respectively. For all area measures, the largest positivity was observed at $\mathrm{Pz}$, intermediate at $\mathrm{Cz}$, and least at Fz, all location comparison $F s(2,33)>14.0, P>0.001$

Separate analyses were conducted in order to investigate the time course of affective responding across the remaining $5 \mathrm{~s}$ of the picture presentation period. These analyses included time (five $1 \mathrm{~s}$ averages) and electrode site (nine) as repeated-measures factors, as well as emotion content. In addition to an overall main effect for valence, $F(2,33)=5.1, P=0.01$, a significant time $\times$ emotion content effect was observed, $F(8,27)=2.4, P<0.05$. Accordingly, the effects of emotion content were assessed separately at each second. As suggested by Fig. 1, analysis of midline leads showed that greater positivity for emotional (pleasant and unpleasant) than neutral pictures was sustained until the fifth second of picture processing: contrast, $F_{S}(1,34)>5.0, P<.025$; emotion content, $F_{S}(2,33)>4.7, P<$ .02 .

For both the analyses of the initial waveform and of the subsequent slow wave, left and right leads showed the same emotional content effects observed at the 
midline. There were neither significant main effects of laterality, nor any interaction of laterality with picture content. As noted earlier, the initial waveform changed significantly in topography from frontal to more posterior electrode sites. Despite this variation, however, the emotional (pleasant and unpleasant) versus neutral picture difference was consistent for all electrodes.

\subsection{Covariation of brain potentials with intensity of emotional activation}

The predominant impression conveyed by the presented results is that viewing emotional pictures, whether they are pleasant or unpleasant, prompts positive voltages at the scalp. Assuming this change reflects affective state, a larger positive slow wave should occur during those pictures, both pleasant and unpleasant, that the participants find more emotionally intense. To assess this hypothesis, ERP waveforms were compared for pictures that participants judged high and low in arousal (median split), within the pleasant and unpleasant valenced contents. Interestingly, these high arousal contents were strong motivational cues, e.g. scenes of violence and erotic images. As can be seen in Table 2, the hypothesis was confirmed: within both emotional valence categories, slow potentials were more positive for the more affectively intense pictures than for lower arousal pictures.

In order to investigate the consistency of this relationship across individuals, correlations were then calculated, for each subject, between the arousal ratings of single picture trials and the mean EEG voltage of the evoked potential in the $700-1000 \mathrm{~ms}$ time window, where overall affect differences were maximal. Of these 35 participants, 33 showed the predicted positive association, and for this small sample of pictures, fully one-third of the correlations were significant (i.e. Pearson

Table 2

Mean difference responses ( $\Delta$ score) of ratings, skin conductance, and EEG onset potentials for pictures high versus low in arousal, separately for pleasant and unpleasant pictures ${ }^{\text {a }}$

\begin{tabular}{lll}
\hline Measures & $\begin{array}{l}\text { Pleasant } \\
\text { pictures }\end{array}$ & $\begin{array}{l}\text { Unpleasant } \\
\text { pictures }\end{array}$ \\
\hline Arousal ratings $(0-20$ scale) & $3.1^{* * *}$ & $2.1^{* * *}$ \\
Valence ratings $(0-20$ scale) & $0.9^{*}$ & $-1.8^{* *}$ \\
Skin conductance response $(\mu \mathrm{S})$ & $0.09^{* * *}$ & 0.025 \\
Averaged ERP amplitude $(\mathrm{Fz}, \mathrm{Cz}, \mathrm{Pz}), 400-700 \mathrm{~ms}(\mu \mathrm{V})$ & $5.9^{* * *}$ & $4.0^{* * *}$ \\
Averaged ERP amplitude $(\mathrm{Fz}, \mathrm{Cz}, \mathrm{Pz}), 700-1000 \mathrm{~ms}(\mu \mathrm{V})$ & $6.4^{* * *}$ & $4.6^{* * *}$
\end{tabular}

a The analyses were repeated using a matched subset of the pictures, such that arousal rating differences were maintained between high and low arousal pictures; however, high and low arousal pictures within pleasant and unpleasant categories did not differ in mean valence rating. The statistical results obtained with the valence-matched pictures were essentially the same as those shown.

$* P<0.05$.

$* * P<0.01$.

*** $P<0.001$. 


\section{Covariation of picture arousal and midline ERP(late positive potential)}

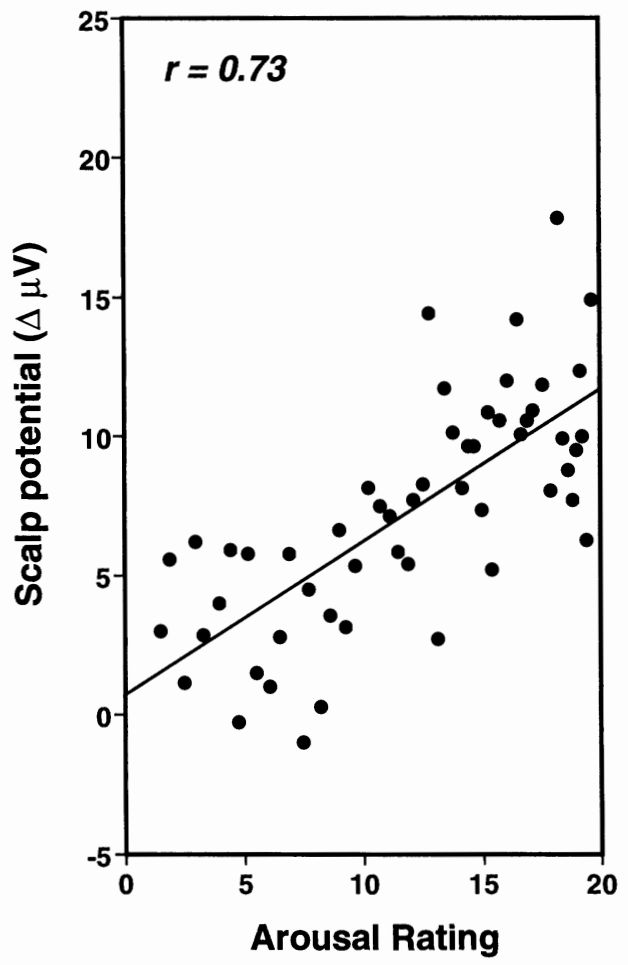

Fig. 2. Covariation of the judged affective arousal of individual pictures and average midline EEG activity in the 700-1000 ms time window. Pictures were rank ordered by arousal judgments for each subject (rank 1-54); the graph shows mean EEG activity based on each individual's response to the picture assigned to that rank.

$r>0.27, \mathrm{df}=53$ ). Ranking all the pictures (pleasant, unpleasant, neutral) according to the SAM arousal judgments of each subject, and averaging across subjects, illustrates the picture by picture relationship between emotional activation and EEG positivity (see Fig. 2).

\subsection{Factor analyses of emotion measures}

Principal component analyses (using varimax solutions) were used to determine how the cortical potential fits into the pattern of emotional responses seen in previous studies of picture perception (for example, Lang et al., 1993). The mean responses across subjects for each of the 54 pictures were used. Thus, individual pictures, rather than subjects, formed the unit of analysis. Factors were retained only if the eigenvalue was larger than 1 . 
The approach paralleled that reported by Lang et al. (1993). In the present case, average EEG activity (Fz, Cz, and $\mathrm{Pz}$ ) in the windows from 400 to 700 and 700 to $1000 \mathrm{~ms}$, means of affective ratings, corrugator responses, heart rate, and skin conductance reactions were entered into the analysis. As shown in Table 3, results replicated and extended the findings reported by Lang et al. (1993). A two-factor solution was obtained, with the factor structure suggesting the names of 'emotional arousal' and 'emotional valence', and accounting for 40 and $30.6 \%$ of the variance, respectively. Both EEG measures, skin conductance response, and arousal judgments loaded on the first factor; corrugator, heart rate, and valence (pleasantness) judgments related to the second factor. A control analysis revealed that entering single electrodes instead of midline-averaged EEG activity resulted in the same factor structure. Extending the input matrix to include vertical eye movement activity (in the same time windows) resulted in a separate third factor, with significant loadings only on the ocular measures.

\section{Discussion}

The results show that emotional pictures prompt a pronounced late positive potential that is greatly reduced or absent for nonaffective stimuli. Furthermore, these positive potentials are specifically enhanced for pictures that are more emotionally intense (i.e. described by viewers as more arousing, and showing a heightened skin conductance response). In previous research, IAPS pictures with similarly high ratings have consistently evoked greater conductance change and have also been judged more 'interesting', been remembered better, and have been looked at longer under free-viewing conditions (see Bradley and Lang (in press) for a review). It is suggested that, because of their motivational significance, emotional stimuli are selected by the brain for sustained attentive processing (Lang et al., 1997). It is hypothesized that the large positive slow wave of the event-related potential indexes this processing.

Table 3

Sorted loadings of dependent measures on principal components, indicating cross-validation with data from Lang et al. (1993) (shown in italics) ${ }^{\mathrm{a}}$

\begin{tabular}{lcc}
\hline Measure & Valence factor & Arousal factor \\
\hline Valence ratings & $\mathbf{0 . 8 7}(\boldsymbol{O . 8 6})$ & $0.06(-0.00)$ \\
Corrugator EMG change & $-\mathbf{0 . 8 2}(-\boldsymbol{0 . 8 5})$ & $-0.11(0.19)$ \\
Heart rate peak & $\mathbf{0 . 7 4}(\mathbf{0 . 7 9})$ & $0.09(-0.14)$ \\
Arousal ratings & $-0.18(0.15)$ & $\mathbf{0 . 8 4}(\boldsymbol{0 . 8 3})$ \\
Averaged ERP amplitude $(400-700 \mathrm{~ms})$ & $0.29(n / a)$ & $\mathbf{0 . 8 6}(\boldsymbol{n} / \boldsymbol{a})$ \\
Averaged ERP amplitude $(700-1000 \mathrm{~ms})$ & $0.19(n / a)$ & $\mathbf{0 . 9 0}(\boldsymbol{n} / \boldsymbol{a})$ \\
Skin conductance response & $0.12(-0.37)$ & $\mathbf{0 . 7 0}(\mathbf{0 . 7 4})$ \\
Interest ratings & n/a $(0.45)$ & $\mathbf{n} / \mathbf{a}(\mathbf{0 . 7 7})$ \\
Viewing time & n/a $(-0.37)$ & $\mathbf{n} / \mathbf{a}(\boldsymbol{O} .74)$ \\
\hline
\end{tabular}

${ }^{\mathrm{a}} \mathrm{n} / \mathrm{a}$, Not applicable. 
Another notable feature of the current results was the extended duration of the slow wave for affective stimuli, maintained until the fifth second of picture viewing. The time course of this response is much longer than typically observed for slow potential onsets to simple tones or lights in single (for example, Simons et al., 1987) or two-stimulus paradigms (for example, see review by Simons (1988)). It seems highly likely that the sustained positivity seen here reflects continued perceptual processing (Kok, 1997). This interpretation is further supported by Schupp et al. (1997). They studied ERPs to brief noise probes, presented between 2.5 and $5 \mathrm{~s}$ after picture onset, distributed over a 6 -s picture presentation period. The magnitude of the P3 to these probes was greater during neutral than emotional pictures. This suggests that attentional resources were devoted to emotional pictures over this extended period. More resources were available for the probe only when the picture did not evoke affect.

Cacioppo et al. $(1993,1994)$ reported late cortical positivity to emotional stimuli (recording from the scalp with a short time constant) that increased with a target's greater 'evaluative distance' from a stimulus context. In their paradigm, the evaluatively distant stimulus was an 'oddball'. In consideration of classic P3 research (Donchin et al., 1986; Donchin and Coles, 1988), the Cacioppo et al. studies raised the possibility that the positivity observed in the present research might be related to internal stimulus probabilities rather than to emotion. The waveforms seen here, however, appear to be unrelated to the 'oddball' effect. If subjects spontaneously categorized the pictures on the basis of valence, all three categories (pleasant, neutral and unpleasant) were equally probable. Alternatively, assuming subjects saw the pictures as forming only two categories (emotional or neutral), the emotional stimuli showing the signature late positivity were actually twice as frequent as the neutral. That is, assuming this unequal, dichotomous classification, the neutral pictures are expected to show a larger P3-like effect, conferred by their 'oddball' status?.

This consideration of local stimulus probability does not, of course, address the problem of real-world probability. For modern civilization, there is an inevitable confound: highly arousing emotional stimuli of all types might be considered less probable than routine day to day input. Recently, Orozco and Ehlers (1998) argued that larger late positive ERPs to erotic pictures by males (than females) supported the hypothesis that this response was mediated by emotional arousal. The unequal distribution by sex in the present sample precluded a successful test of this hypothesis. However, the mean ERP positivity to erotic stimuli shown by males was indeed larger than the mean for females. We might presume that adolescent and just post-adolescent males spend more time looking at erotic pictures than do their

\footnotetext{
${ }^{7}$ In collaboration with John Cacioppo's laboratory (Schupp et al., in press), we recently studied affective picture processing, using brief picture exposure $(1.5 \mathrm{~s})$ and sequences of six pictures, as in the 'evaluative distance' studies. However, participants viewed a balanced group of pleasant, neutral, and unpleasant pictures, without oddball targets. Consistent with the present experiment, and supporting the view that motivational relevance is a primary modulator of the ERP, late positivity was again significantly greater for emotional than neutral pictures.
} 
female peers. If we credit this argument, the real-world probability hypothesis would predict that males would have a diminished rather than a larger late positive ERP. In any event, given the increase in late positive response amplitude from frontal to parietal sites in studies of emotional stimuli, it seems likely that this response depends on the same neural generator that mediates the classical P3 findings. Furthermore, the present data strongly suggest that emotional arousal is considerably more potent in determining late positivity than local probability ${ }^{8}$.

Diedrich et al. (1997) have also reported late positivity to emotional pictures. Furthermore, for subjects instructed to emotionally process the pictures, anterior/ posterior waveform differences were noted: frontal sites showed greater positivity for pleasant than unpleasant pictures, while both emotional contents were similarly more positive than neutral pictures at parietal sites. In the present research, waveform differences between frontal and parietal electrodes were also observed: The frontal response was generally more voltage negative for most of the first second after picture onset. Furthermore, pleasant pictures showed greater positivity, and could be discriminated from neutral pictures earlier in the development of the ERP waveform, than could unpleasant pictures. This effect was not, however, restricted to frontal sites, and later in processing, both types of emotional pictures (pleasant and unpleasant) were significantly more positive than neutral.

This early positive shift for pleasant pictures could reflect a real valence difference at the cortical level of affective processing. Other data from the present experiment suggest, however, that it may be secondary to a chance difference in the emotional intensity of the two picture types. That is, while no difference between pleasant and unpleasant pictures existed for arousal ratings, pleasant pictures did evoke a significantly greater skin conductance response. Skin conductance change is a direct consequence of general activation of the autonomic nervous system, and it is arguably a better measure of biological arousal than subjective evaluation. From this perspective, while the earlier positive potential for pleasant pictures clearly indicates augmented affective arousal (as does the emotional-neutral picture difference), it is not strong evidence for an intrinsic valence difference (i.e. it could be the result of an arousal difference in this particular picture sample). Thus, for example, phobic subjects might show greater earlier positivity for fearful, unpleasant pictures than for either pleasant or neutral stimuli.

Cacioppo's laboratory has reported a larger late positive potential over the right parietal surface, particularly during 'attitudinal categorization' of emotional pictures (for example, Cacioppo et al., 1996), which they relate to an evaluative process. Diedrich et al. (1997) found larger positive potentials when subjects were instructed to process the emotional content of the pictures, compared with a distraction task, but did not report laterality differences specific to pleasant versus

\footnotetext{
${ }^{8}$ Oliver-Rodriguez et al. (1999) reached a similar conclusion in a study of ERP late positivity and gender differences in response to face stimuli, stating that the "construct of emotional value" was "more parsimonious than ... task relevance and subjective probability" in accounting for their ERP late positivity findings (p.183).
} 
unpleasant pictures. Bruder (1995), Davidson (1995) and Heller et al. (1997) have all emphasized electro-cortical differences between the hemispheres in emotional processing and affective style. However, these investigators have focused on assessing EEG frequency in the alpha band, rather than ERPs. In the present experiment, no ERP laterality effects survived statistical analysis. It is possible that future work will uncover reliable hemispheric difference in the evoked potential. Nevertheless, because volume conductance obscures localized effects, the success of this effort will depend on electrode arrays of much higher density than those used here (for example, see Tucker et al., 1994), and continuing improvement in source localization algorithms.

In summary, the present findings show clearly that the brain responds selectively to emotionally evocative pictures and that this reaction increases with reports of greater affective arousal. We suggest that the positive slow potentials indicate increased attentive processing, reflecting activation of motivational systems in the brain, that simultaneously prompt autonomic arousal, emotional facial expression, and reports of affective experience.

\section{Acknowledgements}

This work was supported in part by National Institute of Mental Health grants MH37757, MH43975 and MH52384, to P.J.L.; by the Deutsche Forschungsgemeinschaft (SFB 307, B1); and by a grant from the Deutsche Akademische Auslandsdienst to H.T.S.

\section{References}

Birbaumer, N., Elbert, T., Canavan, A., Rockstroh, B., 1990. Slow potentials of the cerebral cortex and behavior. Physiological Reviews 70, 1-41.

Bradley, M.M., Lang, P.J., 1994. Measuring emotion: the self-assessment manikin and the semantic differential. Journal of Behavior Therapy and Experimental Psychiatry 25, 49-59.

Bradley, M., Lang, P., in press. Measuring emotion: behavior, feeling and physiology. In: Lane, R., Nadel, L. (Eds.), Cognitive Neuroscience of Emotion. Oxford University Press, Oxford (in press).

Bradley, M.M., Cuthbert, B.N., Lang, P.J., 1990. Startle reflex modification: emotion or attention? Psychophysiology 27, 513-521.

Bradley, M.M., Greenwald, M.K., Petry, M., Lang, P.J., 1992. Remembering pictures: pleasure and arousal in memory. Journal of Experimental Psychology 18, 379-390.

Bruder, G., 1995. Cerebral laterality and psychophysiology: perceptual and event-related potential asymmetries in affective and schizophrenic disorders. In: Davidson, R.J., Hugdahl, K. Jr (Eds.), Brain Asymmetry. MIT Press, Cambridge, MA, pp. 661-691.

Cacioppo, J.T., Berntson, G.G., 1994. Relationships between attitudes and evaluative space: a critical review with emphasis on the separability of positive and negative substrates. Psychological Bulletin $115,401-423$.

Cacioppo, J.T., Crites, S.L. Jr, Berntson, G.G., Coles, M.G.H., 1993. If attitudes affect how stimuli are processed, should they not affect the event-related brain potential? Psychological Science 4, 108-112.

Cacioppo, J.T., Crites, S.L. Jr, Gardner, W.L., Berntson, G.G., 1994. Bioelectrical echos from evaluative categorization: I. A late positive brain potential that varies as a function of trait negativity and extremity. Journal of Personality and Social Psychology 67, 115-125. 
Cacioppo, J.T., Crites, S.L. Jr, Gardner, W.L., 1996. Attitudes to the right: evaluative processing is associated with lateralized late positive event-related brain potentials. Personality and Social Psychology Bulletin 6227, 1205-1219.

Center for the Study of Emotion and Attention, 1999. The international affective picture system (IAPS; photographic slides). CSEA-NIMH, University of Florida, Gainesville, FL.

Cook, E.W.I., 1997. VPM Reference Manual. Department of Psychology, University of Alabama, Birmingham, AL.

Cuthbert, B.N., Schupp, H., McManis, M., Hillman, C., Bradley, M.M., Lang, P.J., 1995. Cortical slow waves: emotional perception and processing. Psychophysiology 32, 526.

Davidson, R.J., 1995. Cerebral asymmetry, emotion, and affective style. In: Davidson, R.J., Hugdahl, K. (Eds.), Brain Asymmetry. MIT Press, Cambridge, MA, pp. 361-387.

Diedrich, O., Naumann, E., Maier, S., Becker, G., 1997. A frontal positive slow wave in the ERP associated with emotional slides. Journal of Psychophysiology 11, 71-84.

Donchin, E., Coles, M.G., 1988. Is the P300 component a manifestation of context updating? Behavioral and Brain Sciences 11, 357-427.

Donchin, E., Kramer, A.F., Wickens, C., 1986. Applications of brain event-related potentials to problems in engineering psychology. In: Coles, M.G.H., Donchin, E., Porges, S.W. (Eds.), Psychophysiology - Systems, Processes, and Applications. The Guilford Press, New York, pp. 702-718.

Gratton, G., Coles, M.G., Donchin, E., 1983. A new method for off-line removal of ocular artifact. Electroencephalography and Clinical Neurophysiology 55, 468-484.

Greenwald, M.K., Cook, E.W., Lang, P.J., 1989. Affective judgment and psychophysiological response: dimensional covariation in the evaluation of pictorial stimuli. Journal of Psychophysiology 3, 51-64.

Heller, W., Nitschke, J., Etienne, M., Miller, G., 1997. Patterns of regional brain activity differentiate different types of anxiety. Journal of Abnormal Psychology 106, 376-385.

Hodes, R.L., Cook, E.W., Lang, P.J., 1985. Individual differences in autonomic response: conditioned association or conditioned fear? Psychophysiology 22, 545-560.

Johnston, V.S., Oliver-Rodríguez, J.C., 1997. Facial beauty and the late positive component of event-related potentials. The Journal of Sex Research 34, 188-198.

Johnston, V.S., Miller, D.R., Burleson, M.H., 1986. Multiple P3s to emotional stimuli and their theoretical significance. Psychophysiology 23, 684-693.

Kok, A., 1997. Event-related-potential (ERP) reflections of mental resources: a review and synthesis. Biological Psychology 45, 19-56.

Konorski. J., 1967. Integrative activity of the brain: an interdisciplinary approach. University of Chicago Press, Chicago, IL.

Lang, P.J., 1980. Behavioral treatment and bio-behavioral assessment: computer applications. In: Sidowski, J.B., Johnson, J.H., Williams, T.A. (Eds.), Technology in Mental Health Care Delivery Systems. Ablex Publishing, Norwood, NJ, pp. 119-137.

Lang, P.J., 1989. What are the data of emotion? In: Hamilton, V., Bower, G.H., Frijda, N. (Eds.), Cognitive Perspectives on Emotion and Motivation. Martinus Nijhoff, Boston, MA, pp. 173-191.

Lang, P.J., Bradley, M.M., Cuthbert, B.N., 1990. Emotion, attention, and the startle reflex. Psychological Review 97, 377-395.

Lang, P.J., Greenwald, M.K., Bradley, M.M., Hamm, A.O., 1993. Looking at pictures: affective, facial, visceral, and behavioral reactions. Psychophysiology 30, 261-273.

Lang, P., Bradley, M.M., Cuthbert, B.N., 1997. Motivated attention: affect, activation, and action. In: Lang, P., Simons, R.F., Balaban, M. (Eds.), Attention and Orienting: Sensory and Motivational Processes. Erlbaum Associates, Hillsdale, NJ.

Lang, P.J., Bradley, M.M., Cuthbert, B.N., 1998. Emotion, motivation, and anxiety: brain mechanisms and psychophysiology. Biological Psychiatry 44, 1248-1263.

Lang, P.J., Bradley, M.M., Cuthbert, B.N., 1999. International affective picture system (IAPS): instruction manual and affective ratings. Technical Report A-4, The Center for Research in Psychophysiology, University of Florida.

Mandler, G., Mandler, J.M., Kremen, I., Sholiton, R., 1961. The response to threat: relations among verbal and physiological indices. Psychological Monographs 75 (22).

Miller, G.A., Gratton, G., Yee, C.M., 1988. Generalized implementation of an eye movement correction procedure. Psychophysiology 25, 241-243. 
Naumann, E., Bartussek, D., Diedrich, O., Laufer, M.E., 1992. Assessing cognitive and affective information processing functions of the brain by means of the late positive complex of the event-related potential. Journal of Psychophysiology 6, 285-298.

Oliver-Rodríguez, J.C., Guan, Z., Johnston, V.S., 1999. Gender differences in late positive components evoked by human faces. Psychophysiology 36, 176-185.

Orozco, S., Ehlers, C.L., 1998. Gender differences in electrophysiological responses to facial stimuli. Biological Psychiatry 44, $281-289$.

Palomba, D., Angrilli, A., Mini, A., 1997. Visual evoked potentials, heart rate responses and memory to emotional pictorial stimuli. International Journal of Psychophysiology 27, 55-67.

Ritter, W., Ruchkin, D.S., 1992. A review of event-related potential components discovered in the context of studying P3. In: Friedman, D., Bruder, G.E. (Eds.), Psychophysiology and Experimental Psychopathology: A Tribute to Samuel Sutton. In: Annals of the New York Academy of Sciences, vol. 658. New York Academy of Sciences, New York, pp. 1-32.

Rösler, F., Heil, M., Roeder, B., 1997. Slow negative brain potentials as reflections of specific modular resources of cognition. Biological Psychology 45, 109-141.

Ruchkin, D.S., Johnson, R., Mahaffey, D., Sutton, S., 1988. Toward a functional categorization of slow waves. Psychophysiology 25, 339-353.

Schupp, H.T., Cuthbert, B.N., Bradley, M.M., Birbaumer, Lang, P.J., 1997. Probe P3 and blinks: two measures of affective startle modulation. Psychophysiology 34, 1-6.

Schupp, H.T., Cuthbert, B.N., Bradley, M.M., Cacioppo, J.T., Ito, T., Lang, P.J., (in press). Affective picture processing: the late positive potential is modulated by motivational relevance. Psychophysiology.

Simons, R.F., 1988. Event-related slow brain potentials: a perspective from ANS psychophysiology. In: Ackles, P.K., Jennings, J.R., Coles, M.G.H. (Eds.), Advances in Psychophysiology, vol. 3. JAI Press, Greenwich, CA, pp. 223-267.

Simons, R.F., Rockstroh, B., Elbert, T., Fiorito, E., 1987. Evocation and habituation of autonomic and event-related potential responses in a nonsignal environment. Journal of Psychophysiology 1, 45-59.

Tucker, D., Lotti, M., Potts, B.F., Russel, G.S., Posner, M.I., 1994. Spatiotemporal analysis of brain electrical fields. Human Brain Mapping 1, 134-152. 